\title{
Re-stratification of Patients with High-Risk Prostate Cancer According to the NCCN Guidelines among Patients Who Underwent Radical Prostatectomy: An Analysis Based on the K-CaP Registry
}

\author{
Kwang Suk Lee, $\mathrm{MD}^{1}$ \\ Kyo Chul Koo, MD, PhD' \\ In Young Choi, $\mathrm{PhD}^{2}$ \\ Ji Youl Lee, MD, PhD 3 \\ Jun Hyuk Hong, MD, PhD ${ }^{4}$ \\ Choung-Soo Kim, MD, PhD ${ }^{4}$ \\ Hyun Moo Lee, MD, PhD 5 \\ Sung Kyu Hong, MD, $P h D^{6}$ \\ Seok-Soo Byun, MD, $\mathrm{PhD}^{6}$ \\ Koon Ho Rha, MD, PhD' \\ Byung Ha Chung, MD, PhD ${ }^{1}$
}

${ }^{1}$ Department of Urology, Yonsei University College of Medicine, Seoul,

${ }^{2}$ Graduate School of Management and Policy,

The Catholic University of Korea, Seoul,

${ }^{3}$ Department of Urology, Seoul St. Mary's

Hospital, College of Medicine,

The Catholic University of Korea, Seoul,

${ }^{4}$ Department of Urology,

Asan Medical Center, University of

Ulsan College of Medicine, Seoul,

${ }^{5}$ Department of Urology, Samsung Medical Center, Sungkyunkwan University School of Medicine, Seoul, ${ }^{6}$ Department of Urology,

Seoul National University Bundang Hospital,

Seongnam, Korea

\section{Purpose}

The present study aimed to re-stratify patients with high-risk prostate cancer according to the National Comprehensive Cancer Network guidelines among patients who underwent radical prostatectomy $(\mathrm{RP})$.

\section{Materials and Methods}

This study used the Korean Prostate Cancer Database registry and identified 1,060 patients with high-risk prostate cancer who underwent RP between May 2001 and April 2013. All patients were categorized into risk groups, and subgroups were identified according to the type and number of high-risk factors.

\section{Results}

Of the 1,060 high-risk patients, 599 (56.5\%), 408 (38.5\%), and 53 (5.0\%) had 1, 2, and 3 risk factors, respectively. In multivariate analysis, the Gleason score, percentage of positive biopsy cores, and number of risk factors present were identified as independent predictors of biochemical recurrence. There were significant differences in the 5-year postoperative biochemical failure-free survival (BCFFS) rate among the different high-risk factor subgroups (log-rank $p<0.001$ ). There were no significant differences in the BCFFS rate between the subgroup of high-risk patients with a prostate-specific antigen level $>20 \mathrm{ng} / \mathrm{mL}$ alone and the intermediate-risk group with all factors (log-rank $p=0.919$ and $p=0.781$, respectively). Additionally, no significant difference was noted in the BCFFS rate between high-risk patients having all factors and those in the very-high-risk group ( $p=0.566)$.

\section{Conclusion}

We successfully re-stratified patients with high-risk prostate cancer and identified the combinations of high-risk criteria that will help in the selection of patients for RP.

\section{Key words}

Prostatic neoplasms, Prostatectomy, Risk assessment
Correspondence: Byung Ha Chung, MD, PhD Department of Urology, Yonsei University College of Medicine, 50-1 Yonsei-ro, Seodaemun-gu, Seoul 03722, Korea Tel: 82-2-2019-3470

Fax: 82-2-3462-8887

E-mail: chung646@yuhs.ac

Received October 15, 2016 Accepted February 28, 2017

Published Online March 7, 2017 


\section{Introduction}

Prostate cancer $(\mathrm{PCa})$ has been reported to be the most common malignancy among elderly men in Western countries [1,2]. In Asia, the incidence and detection of PCa have recently increased. Several factors, including environmental factors, changes in Western dietary habits, medical advances in laboratory diagnosis, and campaigns for prostate-specific antigen (PSA) screening, are believed to be associated with the increased incidence of PCa [3]. A previous study has reported that many patients present with locally advanced disease [4].

Several treatment options, including radical prostatectomy $(\mathrm{RP})$, radiation therapy (RT), androgen deprivation therapy (ADT), and multidisciplinary therapy, have been recommended for patients with high-risk PCa. Most previous studies that assessed the efficacy of RP, RT, or multidisciplinary therapy for patients with high-risk PCa have demonstrated extremely heterogeneous outcomes according to treatment modality [1,5-8], but there is no data from a multicenter database of high-risk patients with RP. Among patients at high risk of failure following standard therapy, RP allows for treatment individualization by carefully selecting patients who might benefit from treatment. Therefore, it is necessary to revisit the classification system and attempt to better stratify patients within this heterogeneous high-risk PCa group.

There is no consensus on the definition of high-risk PCa $[1,5,9,10]$. In practice, patients with PCa are treated based on the recommendations of the National Comprehensive Cancer Network (NCCN) guidelines. Patients with intermediate and high-risk PCa with multiple adverse factors may be shifted to the next highest risk subgroup [9]. Recently, the NCCN guidelines recommended that patients with high-risk PCa (T3a or Gleason score 8-10 or PSA $>20 \mathrm{ng} / \mathrm{mL}$ ) with primary Gleason pattern 5 or more than 4 cores with Gleason score 8 to 10 should be classified as very high-risk patients, with RP recommended for select patients. Using this classification system, urologists can predict oncological outcomes, including biochemical recurrence (BCR) after definitive therapy, and select appropriate candidates for RP from among patients with high-risk PCa. The present study aimed to further classify a large cohort of patients with high-risk PCa who underwent RP according to the NCCN guidelines.

\section{Materials and Methods}

The study used the Korean Prostate Cancer Database (K-CaP) registry. The K-CaP registry was established in 2014 as the first registry for comprehensive data collection with regard to Korean patients with PCa who underwent RP [11]. Patients with PCa who underwent RP at five Korean tertiary referral hospitals (Asan Medical Center, Samsung Medical Center, Seoul National University Bundang Hospital, Seoul St. Mary's Hospital, and Yonsei University Severance Hospital) between 2001 and 2012 were eligible for inclusion in the $\mathrm{K}-\mathrm{CaP}$ registry. The $\mathrm{K}-\mathrm{CaP}$ registry included a total of 3,206 patients. Patients with a PSA level $>20 \mathrm{ng} / \mathrm{mL}$, a Gleason score of $8-10$ on prostate biopsy, or a clinical stage of T3a using prostate magnetic resonance imaging (MRI) were considered to have high-risk PCa according to the 2016 NCCN guidelines [9]. After excluding patients who received neoadjuvant ADT and those without complete clinicopathological data, 1,060 patients with high-risk PCa were finally enrolled in this study.

The MRI data of the enrolled patients were interpreted by radiologists in the urology department at each participating hospital. The final pathology was determined using RP specimens and was reported by the pathologists in the urology department of each institution. The clinicopathological stage was assigned according to the 2002 TNM staging system, and prostate biopsy cores were obtained with transrectal ultrasound guidance using a $>10$-core biopsy protocol. An adverse pathologic feature was defined as the occurrence of pathologic stage $\geq \mathrm{T} 3 \mathrm{~b}$ cancer. The percent of positive biopsy cores as a continuous variable was defined as the positive core count divided by the total core count.

Clinicopathological data, such as age at surgery, previous medical history, PSA level at diagnosis, prostate size on preoperative imaging studies, prostate biopsy-related variables, operative method, and pathological outcomes (pathological stage, Gleason score, and surgical margin status), were extracted from the $\mathrm{K}-\mathrm{CaP}$ registry. Biochemical failure (BCF) was defined as (1) a confirmed rise in the PSA level above the threshold of $0.2 \mathrm{ng} / \mathrm{mL},(2)$ a failure of PSA to fall to undetectable levels, or (3) the use of additional therapy including RT or ADT.

This study was performed after obtaining approval from the Institutional Review Board (2016-0493-001). The patient and tumor characteristics were compared using Pearson's chi-square test and Student's t test. Cox univariate and multivariate analyses were performed to assess the clinical variables predictive of BCF in all patients with high-risk PCa. The postoperative BCF-free survival (BCFFS) rate of each patient group was calculated and compared using Kaplan-Meier analysis with a log-rank test. All statistical analyses were performed using SPSS ver. 21 (IBM Corp., Armonk, NY). A $\mathrm{p}$-value of $<0.05$ was considered to indicate a statistically significant difference. 


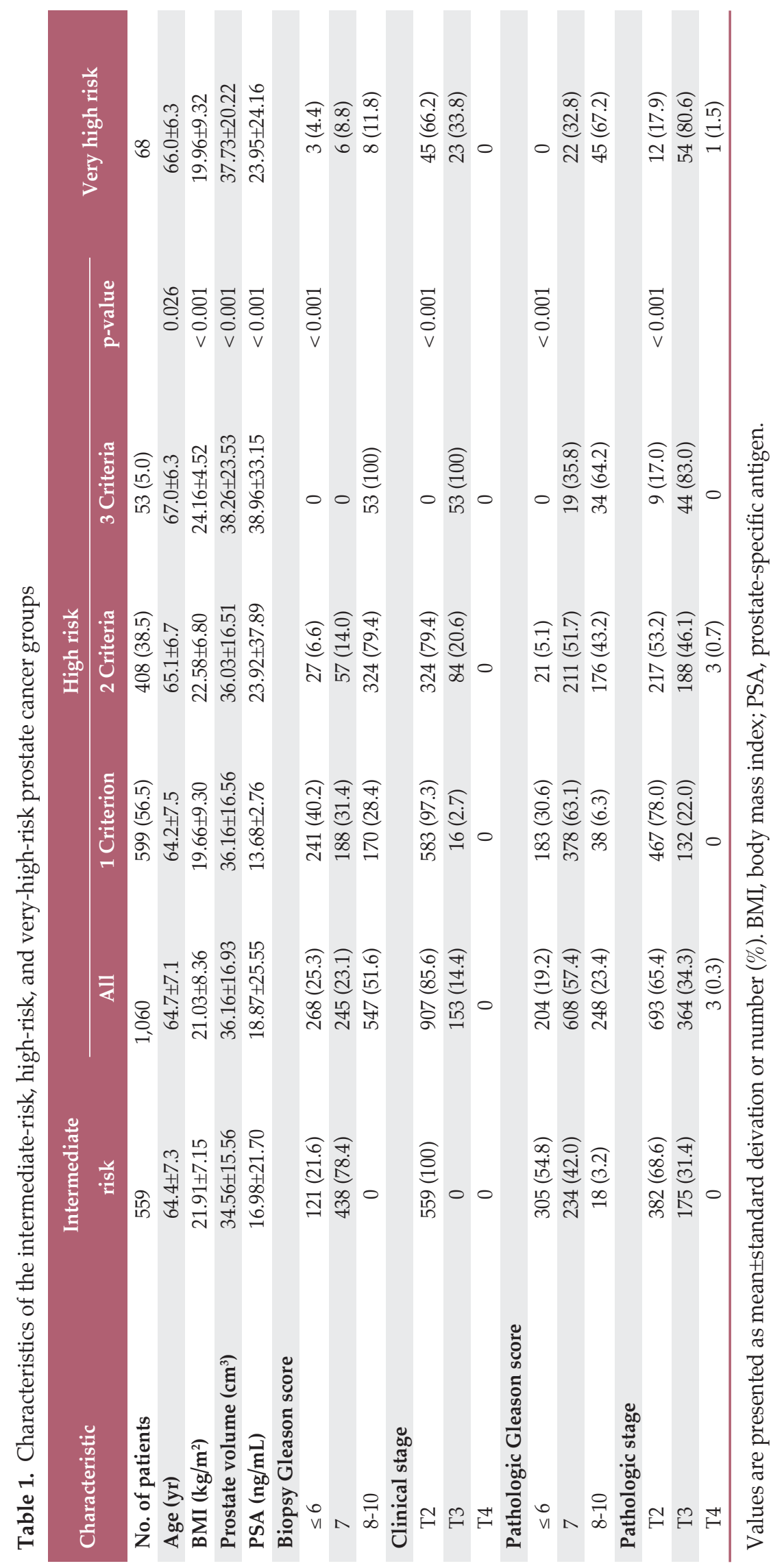


Table 2. Univariate and multivariate analyses for the identification of the significant predictors of postoperative biochemical failure in the high-risk group

\begin{tabular}{|c|c|c|c|c|}
\hline \multirow{2}{*}{ Variable } & \multicolumn{2}{|c|}{ Univariate analysis } & \multicolumn{2}{|c|}{ Multivariate analysis } \\
\hline & Hazard ratio $(95 \% \mathrm{CI})$ & p-value & Hazard ratio $(95 \% \mathrm{CI})$ & p-value \\
\hline Age $(y r)^{a)}$ & $1.01(0.996-1.027)$ & 0.148 & - & - \\
\hline BMI $\left(<25 \mathrm{~kg} / \mathrm{m}^{2} \mathrm{vs} . \geq 25 \mathrm{~kg} / \mathrm{m}^{2}\right)$ & $1.04(0.890-1.207)$ & 0.641 & - & - \\
\hline Prostate volume $\left(\mathrm{cm}^{3}\right)^{\mathrm{a})}$ & $1.00(0.991-1.001)$ & 0.081 & - & - \\
\hline $\operatorname{PSAD}\left(\mathrm{ng} / \mathrm{mL} / \mathrm{cm}^{3}\right)^{\mathrm{a})}$ & $1.59(1.440-1.764)$ & $<0.001$ & $1.33(1.162-1.515)$ & $<0.001$ \\
\hline PSA (> $20 \mathrm{ng} / \mathrm{mL}$ vs. $20 \mathrm{ng} / \mathrm{mL}$ ) & - & $<0.001$ & $1.66(1.355-2.030)$ & $<0.001$ \\
\hline Stage (T3a vs. $\leq \mathrm{T} 2 \mathrm{c}$ ) & $2.86(2.325-3.524)$ & $<0.001$ & $1.51(1.112-2.036)$ & $<0.001$ \\
\hline Gleason score ( $\geq 8$ vs. $\leq 7$ ) & $2.83(2.422-3.298)$ & $<0.001$ & $2.00(1.623-2.461)$ & $<0.001$ \\
\hline Positive biopsy core (\%) ${ }^{\mathrm{a})}$ & $1.14(1.111-1.178)$ & $<0.001$ & $1.93(1.333-2.778)$ & $<0.001$ \\
\hline
\end{tabular}

CI, confidence interval; BMI, body mass index; PSAD, prostate-specific antigen density; PSA, prostate-specific antigen. ${ }^{\text {a) }}$ Continuous variable.

\section{Results}

Table 1 presents the baseline characteristics of the intermediate-risk, high-risk, and very-high-risk PCa groups based on the NCCN guidelines. Of the 1,060 patients with high-risk PCa included in this study, open RP, laparoscopic RP, and robotassisted laparoscopic prostatectomy were performed in 359 $(33.9 \%), 17(1.6 \%)$, and $684(64.5 \%)$ patients, respectively. The median duration of postoperative follow-up was 48.8 months (interquartile range, 32.0 to 60.0 months). The number of patients with 1, 2, and 3 risk factors was 599 (56.5\%), 408 (38.5\%), and $53(5.0 \%)$, respectively. According to the risk classification, the mean PSA level was $15.68 \mathrm{ng} / \mathrm{mL}$ for one risk factor, $23.92 \mathrm{ng} / \mathrm{mL}$ for two risk factors, and $38.96 \mathrm{ng} / \mathrm{mL}$ for three risk factors, respectively $(\mathrm{p}<0.001)$. The proportions of the clinical stage were different between the three groups ( $\leq \mathrm{T} 2 \mathrm{a}, 72.4 \%, 45.9 \%$, and $0 \%$; cT2b-cT2c, $14.5 \%, 33.4 \%$ and $0 \%$; cT3a, $13.0 \%, 20.6 \%$, and $100.0 \%$ for 1,2 , and 3 risk factors, respectively [ $p<0.001])$. There were significant differences in the rates of adverse pathological features among the different high-risk factor subgroups ( 1 vs. 2 vs. 3, 3.5\% vs. $19.1 \%$ vs. $20.8 \% ; \mathrm{p}=0.001)$. The proportion of perineural invasion increased with the number of risk factors ( 1 vs. 2 vs. $3,50.6 \%$ vs. $63.0 \%$ vs. $83.0 \%$; $\mathrm{p}=0.001$ ).

In the multivariate Cox proportional hazards models, including age, body mass index, PSA level (>20 ng/ mL vs. 20 $\mathrm{ng} / \mathrm{mL}$ ), PSA density, stage (T3a vs. $\leq \mathrm{T} 2 \mathrm{c}$ ), Gleason score ( $\geq 8$ vs. $\leq 7)$, and percent of positive biopsy cores, we found that PSA level, PSA density, stage, Gleason score, and percent of positive biopsy cores were independent predictors of $\mathrm{BCF}$ in the high-risk PCa group. Of high-risk factors, the strongest predictor of progression and mortality was the Gleason score

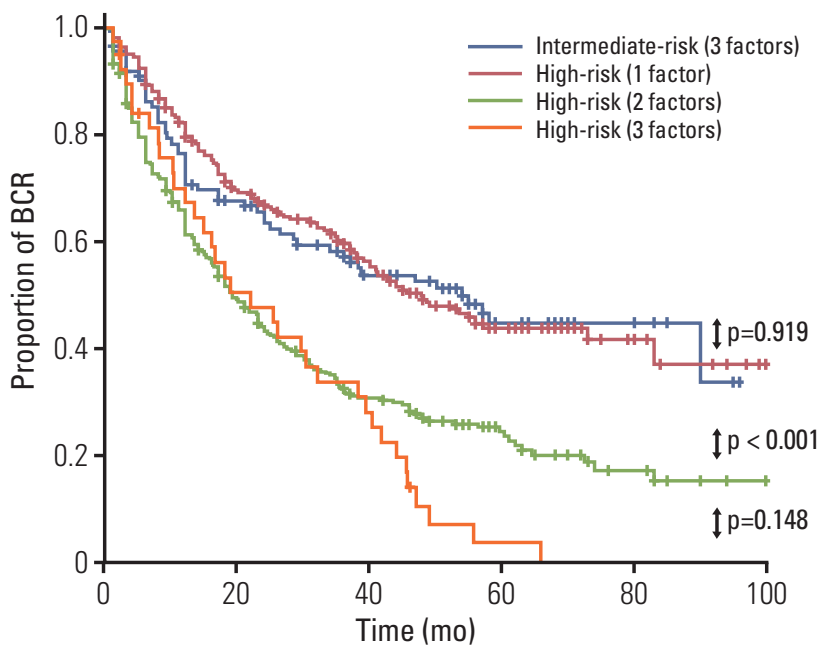

Fig. 1. Biochemical recurrence $(B C R)$ in the very-low-risk, low-risk, intermediate-risk, high-risk, very-high-risk, and metastatic-risk groups.

(Table 2). We evaluated the comparative survival of and number of high-risk factors ( $\geq 2$ vs. 1) after adjustment for covariates considered potential predictors by the Cox proportional hazards analysis for BCF (hazard ratio, 1.36; 1.016 to 1.826; $\mathrm{p}=0.039$ ).

We found significant differences in the 5-year postoperative BCFFS rate among the different high-risk factor subgroups ( 1 vs. 2 vs. $3,43.0 \%$ vs. $21.0 \%$ vs. $0 \%$; log-rank p < 0.001 ). However, there was no significant difference in the BCFFS rate between the high-risk group with one factor and the intermediate-risk group with all factors (log-rank $\mathrm{p}=0.919)$ (Fig. 1). On 
further analyzing the high-risk group with one factor, we found that the BCFFS rate was similar between the subgroup of patients with a PSA level $>20 \mathrm{ng} / \mathrm{mL}$ alone and the intermediate-risk group with all factors (log-rank $\mathrm{p}=0.781, \mathrm{p}=0.001$, and $\mathrm{p}<0.001$ for the factors, respectively). On comparing biochemical outcomes between patients having all high-risk factors and those in the very-high-risk group, no significant difference was found in the BCFFS rate (log-rank $p=0.566$ ).

\section{Discussion}

In the present study, we observed heterogeneous pathological and biochemical outcomes among patients who underwent RP for high-risk PCa according to the NCCN guidelines [9]. We found that BCFFS was related to the number of high-risk factors. This study will help in the selection of patients for RP using combinations of present high-risk criteria. Overall, our findings support the accurate characterization of patients with high-risk PCa according to the new NCCN risk stratification.

The main considerations when deciding on PCa treatment are the risk factors, including the PSA level, TNM clinical stage, Gleason score, age, and comorbidities. At present, urologists face the dilemma of deciding which treatment is best adapted for patients with high-risk PCa: RP, RT, or multidisciplinary therapy. Several studies have assessed risk stratification using different approaches, such as the Kattan nomogram and Cancer of the Prostate Risk Assessment $[12,13]$. With regard to digital rectal examination and computed tomography, clinical staging can be imprecise for the evaluation of extraprostatic disease. MRI is believed to improve the accuracy of clinical staging. In our study, all patients were stratified into new risk groups according to the NCCN guidelines using prostate MRI for precise clinical staging.

Clinically useful criteria for defining high-risk PCa should reliably distinguish patients who might be optimal candidates for RP. Prospective randomized trials have been performed to provide insight into treatment strategies; however, the optimal strategy has not been definitively identified [14-16]. The NCCN guidelines suggest criteria that divide patients with high-risk $\mathrm{PCa}$ into various groups according to distinct clinical outcomes. The very-high-risk group is defined by the following criteria at diagnosis: a primary Gleason pattern of 5 or $>4$ cores with a Gleason score of 8-10 and clinical stage T3b-4. Therefore, there have been no previous studies with a proper target patient group.

High-risk patients are generally at an elevated risk for early $\mathrm{BCR}$, metastatic progression, and death from $\mathrm{PCa}$
$[10,17,18]$. Therefore, a consensus on the definition of highrisk PCa is considered a priority [3]. Yossepowitch et al. [17] reported that the 5-year biochemical recurrence-free survival rate ranged from $49 \%$ to $80 \%$ according to eight different definitions of high-risk PCa. In this study, a PSA level $>20$ $\mathrm{ng} / \mathrm{mL}$, stage CT3, and a positive biopsy core with a Gleason score of 8-10 individually or in combination were considered factors associated with high-risk disease. Joniau et al. [19] compared PCa-specific survival using seven different definitions of high-risk PCa to classify 1,632 patients treated with RP. The authors suggested stratification of patients with high-risk PCa into the following three subgroups: good prognosis subgroup (1 high-risk factor); intermediate prognosis subgroup (PSA level $>20 \mathrm{ng} / \mathrm{mL}$ and stage cT3-4); and poor prognosis subgroup (Gleason score 8-10 in combination with at least 1 other high-risk factor). Because of the lack of a definition of high-risk $\mathrm{PC}$, studies have subclassified high-risk PCa into different prognostic categories [20,21].

An elevated PSA level (> $20 \mathrm{ng} / \mathrm{mL}$ ) alone is not enough to define a high-risk patient. Spahn et al. [22] reported that the strongest predictor of prostatectomy was the Gleason score in patients with PSA $>20 \mathrm{ng} / \mathrm{mL}$, and the risk levels affected disease progression and PCa-specific mortality. We found that the BCFFS rate was not different between patients having 1 high-risk factor (PSA level $>20 \mathrm{ng} / \mathrm{mL}$ ) and patients in the intermediate-risk group having all factors. With regard to the PSA level, there can be fluctuations that result from several factors, including benign prostatic hyperplasia and other nonmalignant conditions. Considering that many individuals present with PSA $>20 \mathrm{ng} / \mathrm{mL}$ as their only highrisk factor, they may present with favorable pathologic characteristics and could be appropriate candidates for surgery.

As expected, pathologic features and progression were significantly different among the three risk categories. In patients with one high-risk factor, heterogeneous pathological and biochemical outcomes were found. Patients whose only high-risk factor was an elevated PSA level $(>20 \mathrm{ng} / \mathrm{mL})$ had a similar prognosis to the intermediate-risk group of patients with all factors. The BCFFS rate was not different between patients with three high-risk factors and patients in the very-high-risk group. Our results are consistent with the recommendation of the NCCN guidelines that the intermediate-risk group and patients with high-risk PCa with multiple adverse factors may be shifted to the next risk group. For patients with high-risk PCa whose only risk factor is elevated PSA level (> $20 \mathrm{ng} / \mathrm{mL}$ ), we can recommend $\mathrm{RP}$ as the initial therapy in accordance with the intermediate-risk group, and the high-risk groups with all risk factors should be considered for multidisciplinary therapy instead of RP. In conclusion, the clinician could schedule patients already treated with RP and pelvic lymph node dissection for appropriate postoperative management. $\mathrm{K}-\mathrm{CaP}$ is currently design- 
ing future studies to evaluate whether early postoperative treatments should consequently be reserved for cases of BCF.

Our study has important strengths. To our knowledge, this is the first study to provide consistent predictions of appropriate oncological endpoints in patients presenting with high-risk PCa, based on the number and precise combination of risk factors. Few previous studies have described the outcomes of surgery in patients with high-risk PCa, and most of these studies have presented a small series or a single-center experience. Specifically, after extracting unfavorable factors, including a primary Gleason pattern of 5 or $>4$ cores with a Gleason score of 8-10, from the high-risk factors to identify a group of men within the current NCCN highrisk category who are at greatest risk of metastasis or cancerrelated death despite conventional treatment [23]. our study indicated which patients may require surgery and which patients may not require surgery. Most previous studies aimed to validate the subset of the high-risk group according to the oncological outcomes within each risk group [20,24, 25]. The present study identified the oncological outcomes of each subgroup based on the number of risk factors after comparison with those in the other risk groups in patients with high-risk PCa. Furthermore, there were three subgroups according to the number of high-risk factors, and the outcomes, including BCFFS, were compared to those for the next lowest and highest groups.

Although this study had several strengths, there were some limitations. Several factors could account for the heterogeneity in the results, including the presence of multiple physicians and the variability of intraoperative management. Additionally, there was heterogeneity of the treated patients. Multiple factors influenced the clinical decision-making, including age, performance status, and preoperative clinico- pathological results. In particular, the data on comorbidity that may have affected treatment policies were not perfect. In addition, because of factors such as comorbidities, the prognosis of patients who received other treatments was not known. In our retrospective cohort, all MRI and pathological results were not centrally investigated but were assessed by radiologists and pathologists in the urology department at each institution. Another limitation is the relatively short follow-up period for patients treated with RP, which did not allow analysis of metastasis-free survival or cancer-specific survival. For further survival evaluation, the follow-up period should be longer, and more multicenter trials are needed to clarify whether the oncological outcomes are similar between a high-risk group with a PSA level $>20 \mathrm{ng} / \mathrm{mL}$ and an intermediate-risk group with all risk factors. Moreover, it may not be possible to extend our finding of a similar BCFFS rate between patients having all high-risk factors and patients in the very-high-risk group to high-risk patients undergoing radiotherapy or systemic therapy.

In the present study, we successfully re-stratified patients with high-risk PCa and noted that the use of combinations of present high-risk criteria will help in the selection of patients for RP. Additionally, we noted heterogeneity in biochemical outcomes among patients with high-risk PCa who underwent RP. The postoperative BCFFS rate appears to be similar between patients having 1 high-risk factor (PSA > 20 $\mathrm{ng} / \mathrm{mL}$ ) and patients in the intermediate-risk group and between patients having three high-risk factors and patients in the very-high-risk group.

\section{Conflicts of Interest}

Conflict of interest relevant to this article was not reported.

\section{References}

1. Heidenreich A, Bastian PJ, Bellmunt J, Bolla M, Joniau S, van der Kwast T, et al. EAU guidelines on prostate cancer. part 1: screening, diagnosis, and local treatment with curative intentupdate 2013. Eur Urol. 2014;65:124-37.

2. Cancer statistics. JAMA. 2013;310:982.

3. Chung BH. The role of radical prostatectomy in high-risk prostate cancer. Prostate Int. 2013;1:95-101.

4. Oh CM, Won YJ, Jung KW, Kong HJ, Cho H, Lee JK, et al. Cancer statistics in Korea: incidence, mortality, survival, and prevalence in 2013. Cancer Res Treat. 2016;48:436-50.

5. Carter HB, Albertsen PC, Barry MJ, Etzioni R, Freedland SJ, Greene KL, et al. Early detection of prostate cancer: AUA guideline. J Urol. 2013;190:419-26.
6. Muralidhar V, Chen MH, Reznor G, Moran BJ, Braccioforte $\mathrm{MH}$, Beard CJ, et al. Definition and validation of "favorable high-risk prostate cancer": implications for personalizing treatment of radiation-managed patients. Int J Radiat Oncol Biol Phys. 2015;93:828-35.

7. Nguyen PL, Chen MH, Catalona WJ, Moul JW, Sun L, D'Amico AV. Predicting prostate cancer mortality among men with intermediate to high-risk disease and multiple unfavorable risk factors. Int J Radiat Oncol Biol Phys. 2009;73:659-64.

8. Kobayashi T, Kimura T, Lee C, Inoue T, Terada N, Kono Y, et al. Subclassification of high-risk clinically organ-confined prostate cancer for early cancer-specific mortality after radical prostatectomy. Jpn J Clin Oncol. 2016;46:762-7. 
9. Mohler JL, Armstrong AJ, Bahnson RR, D'Amico AV, Davis BJ, Eastham JA, et al. Prostate cancer, version 1.2016. J Natl Compr Canc Netw. 2016;14:19-30.

10. D'Amico AV, Whittington R, Malkowicz SB, Schultz D, Blank $\mathrm{K}$, Broderick GA, et al. Biochemical outcome after radical prostatectomy, external beam radiation therapy, or interstitial radiation therapy for clinically localized prostate cancer. JAMA. 1998;280:969-74.

11. Lee DH, Lee SH, Rha KH, Choi IY, Lee JY, Kim SW, et al. The establishment of K-CaP (the Multicenter Korean Prostate Cancer Database). Korean J Urol. 2013;54:229-33.

12. Kattan MW, Eastham JA, Stapleton AM, Wheeler TM, Scardino PT. A preoperative nomogram for disease recurrence following radical prostatectomy for prostate cancer. J Natl Cancer Inst. 1998;90:766-71.

13. Cooperberg MR, Pasta DJ, Elkin EP, Litwin MS, Latini DM, Du Chane J, et al. The University of California, San Francisco Cancer of the Prostate Risk Assessment score: a straightforward and reliable preoperative predictor of disease recurrence after radical prostatectomy. J Urol. 2005;173:1938-42.

14. Bolla M, van Poppel H, Tombal B, Vekemans K, Da Pozzo L, de Reijke TM, et al. Postoperative radiotherapy after radical prostatectomy for high-risk prostate cancer: long-term results of a randomised controlled trial (EORTC trial 22911). Lancet. 2012;380:2018-27.

15. Wiegel T, Bottke D, Steiner U, Siegmann A, Golz R, Storkel S, et al. Phase III postoperative adjuvant radiotherapy after radical prostatectomy compared with radical prostatectomy alone in pT3 prostate cancer with postoperative undetectable prostate-specific antigen: ARO 96-02/ AUO AP 09/95. J Clin Oncol. 2009;27:2924-30.

16. Dorff TB, Flaig TW, Tangen CM, Hussain MH, Swanson GP, Wood DP Jr, et al. Adjuvant androgen deprivation for highrisk prostate cancer after radical prostatectomy: SWOG S9921 study. J Clin Oncol. 2011;29:2040-5.

17. Yossepowitch O, Eggener SE, Bianco FJ Jr, Carver BS, Serio A,
Scardino PT, et al. Radical prostatectomy for clinically localized, high risk prostate cancer: critical analysis of risk assessment methods. J Urol. 2007;178:493-9.

18. Yossepowitch O, Eggener SE, Serio AM, Carver BS, Bianco FJ Jr, Scardino PT, et al. Secondary therapy, metastatic progression, and cancer-specific mortality in men with clinically highrisk prostate cancer treated with radical prostatectomy. Eur Urol. 2008;53:950-9.

19. Joniau S, Briganti A, Gontero P, Gandaglia G, Tosco L, Fieuws $S$, et al. Stratification of high-risk prostate cancer into prognostic categories: a European multi-institutional study. Eur Urol. 2015;67:157-64.

20. Jo JK, Kook HR, Byun SS, Lee SE, Hong SK. Stratification of contemporary patients undergoing radical prostatectomy for high-risk prostate cancer. Ann Surg Oncol. 2015;22:2088-93.

21. Walz J, Joniau S, Chun FK, Isbarn H, Jeldres C, Yossepowitch $\mathrm{O}$, et al. Pathological results and rates of treatment failure in high-risk prostate cancer patients after radical prostatectomy. BJU Int. 2011;107:765-70.

22. Spahn M, Joniau S, Gontero P, Fieuws S, Marchioro G, Tombal $\mathrm{B}$, et al. Outcome predictors of radical prostatectomy in patients with prostate-specific antigen greater than $20 \mathrm{ng} / \mathrm{ml}$ : a European multi-institutional study of 712 patients. Eur Urol. 2010;58:1-7.

23. Sundi D, Wang VM, Pierorazio PM, Han M, Bivalacqua TJ, Ball MW, et al. Very-high-risk localized prostate cancer: definition and outcomes. Prostate Cancer Prostatic Dis. 2014;17: 57-63.

24. Reese AC, Pierorazio PM, Han M, Partin AW. Contemporary evaluation of the National Comprehensive Cancer Network prostate cancer risk classification system. Urology. 2012;80: 1075-9.

25. Lughezzani G, Lazzeri M, Haese A, McNicholas T, de la Taille A, Buffi NM, et al. Multicenter European external validation of a prostate health index-based nomogram for predicting prostate cancer at extended biopsy. Eur Urol. 2014;66:906-12. 